\title{
Dynamics of a trapped vortex in rotating convection
}

\author{
J. W. Atkinson, ${ }^{*}$ P. A. Davidson, ${ }^{\dagger}$ and J. E. G. Perry ${ }^{\ddagger}$ \\ Engineering Department, University of Cambridge. \\ Trumpington Street, Cambridge, CB2 1PZ, UK
}

(Dated: June 14, 2019)

We consider axisymmetric rotating convection in a cylindrical domain, focussing on the eye that can form at the centre of a cyclonic vortex. Upon increasing the thermal forcing we observe that the the system undergoes a Hopf bifurcation from a state with a steady eye to one in which the eye oscillates. For an aspect ratio, Ekman number, and Prandtl number of 0.1 we find that the critical Reynolds number at which this transition occurs is 398.

We examine the nature of the oscillations and propose that the behaviour results from an inertial wave trapped in the eye, with the frequencies falling within the expected range for inertial waves, and the oscillations displaying clear similarities to a standing inertial wave in a cylinder. We also examine the effect of Ekman number on the oscillation, finding that there is upper limit beyond which oscillations do not occur.

\footnotetext{
* jwa34@cam.ac.uk

† pad3@cam.ac.uk

¥ jamesegperry@cantab.net
} 


\section{INTRODUCTION}

The subject of eye formation - the development of a region of calm, reversed (or subsiding) flow, localised to the central axis of a vortex - is a particularly interesting problem. It has attracted the attention of geophysical fluid dynamicists for a long time, with an early discussion by Morton [1]. The phenomenon is perhaps most widely recognised in the weather system known as the tropical cyclone, but similar flows have also been observed in columnar vortices such as dust devils [2] and waterspouts [3], and perhaps also tornadoes [4]. A review of the nature and dynamics of the tropical cyclone, including some discussion of eyes, is given by Emanuel [5].

Though many observations have been made of the nature of the eye in the tropical cyclone, there is still a lack of fundamental understanding as to how or why it forms. A number of different theories have been presented to explain the phenomenon and the topic is still strongly debated - see the exchange of Pearce [6, 7] and Smith [8]. Smith [9] suggests that subsiding flow is driven by an axial pressure gradient at the centre of the cyclone imposed by radial pressure gradients due to the swirling flow, a theory that he states complements the ideas of Willoughby [10]. Pearce [11] considers a rather complex model with the origins of the eye explained in a different way. He suggests that the eye is formed as a result of azimuthal vorticity generation due to axial gradients in angular momentum. There are other theories besides the ones singled out here, each with a different explanation and focusing on different aspects of a complex system.

Looking beyond the tropical cyclone literature, similar results have been observed for columnar vortices by Harlow and Stein [12] and Rotunno [13, 14]. They find that numerical investigations of intense, swirling, cylindrical flow can lead to a region of reversed flow at the axis. Harlow and Stein [12] adopt a similar explanation to Smith [9], suggesting that this arises due to an axial pressure gradient imposed by the rotating flow. Rotunno [13] takes an approach closer to that of Pearce [11]. He identifies the eyewall (or 'core wall' as he calls it) as an intense annulus of negative azimuthal vorticity generated by axial gradients in angular momentum. The vortex core enclosed by this annulus then develops a downwards flow in response to the azimuthal vorticity. At intense levels of swirl with a no-slip lower boundary a disruption occurs near the base of the vortex akin to the vortex breakdown observed in experiments [15]. Rotunno notes that when this occurs the eyewall above the breakdown displays perturbations, likely a result of standing centrifugal waves.

It is interesting to note that in some cases Harlow and Stein [12] observe oscillations of the eye. Though remarked upon, they offer no discussion or explanation for this. Oscillations have also been observed in models of dust devils by Castaño et al. [16]. In this case the oscillations are asymmetric and occur through a sequence of Hopf bifurcations. Oscillations have also been recorded in tropical cyclones by Chen et al. [17] who provide a description of high frequency oscillations observed within the eye. These oscillations display similarities to those reported by Harlow and Stein [12] with fluctuations of the vertical velocities within the eye.

One omission in much of the work of Harlow and Stein [12], Rotunno [13], and the model of Pearce [11] is the presence of a no-slip lower boundary. This is discussed in a recent paper by Oruba et al. [18]. They present a purely hydrodynamical explanation for the formation of eyes in atmospheric vortices. Like Pearce [11] and Rotunno [13], they propose that the eye is formed as a passive response to the development of an inverted conical region of intense negative azimuthal vorticity, the so-called eyewall. The key difference between this and previous works, however, is the origin of this negative azimuthal vorticity (see figure 1). Whilst others suppose that it arises due to axial gradients in angular momentum, Oruba et al. [18] point out that these axial gradients can make no net contribution to the azimuthal vorticity. The negative vorticity that comprises the eyewall is in fact generated in the lower boundary layer. To test this hypothesis they perform numerical simulations of a simplified model problem; rotating convection in a shallow basin with a no-slip base. They observe a secondary meridional flow, spun up by the Coriolis force, consisting of a single large convective cell. The vorticity that is generated at the lower boundary is swept upwards as the flow converges at the central axis to produce an eyewall and then, provided the eyewall is strong enough, an eye. They found that the flow was largely dependent on Reynolds number (and hence the temperature difference between the top and bottom of the vortex), with only a slight dependence on Prandtl number. The dependencies on aspect ratio, Ekman number (rotation rate) and Prandtl number (thermal diffusivity) were explored further in a follow up paper [19]. A detailed discussion of the dynamics of the tropical cyclone boundary layer, including how it may be swept into the eyewall, is given by Smith and Montgomery [20].

Many fluid systems undergo bifurcations in flow structure or behaviour as the forcing is increased. Canonical examples are Taylor-Couette flow [21], and Rayleigh-Bérnard convection [22]. The nature of bifurcations in fluid mechanics is well discussed by Benjamin [23]. We observe that the cyclone transitions from a vortex with a steady eye to a state in which the eye oscillates as the thermal forcing is increased, eventually displaying chaotic motion.

This paper further explores the model proposed by [18]. After an overview of the model and the governing equations in section II, we present numerical simulations, the details of which are given in section III. The first results in section IV concern the formation of a steady eye. We follow this with our observations of oscillatory eyes in section V. After characterising the nature of the oscillation we finally consider the possibility that this phenomenon can be explained 


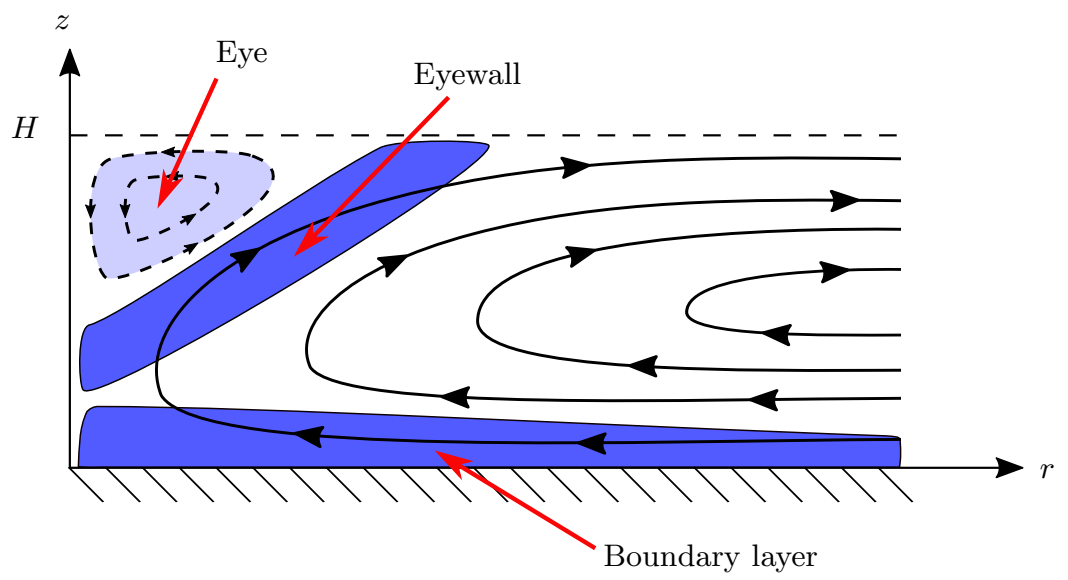

FIG. 1: Sketch of the eye, eyewall, and lower boundary layer.

by the presence of a trapped inertial wave in section VI.

\section{GOVERNING EQUATIONS AND MODEL PROBLEM}

Like Oruba et al. [18], we consider a shallow, cylindrical $(r, \phi, z)$, axisymmetric, domain of height $H$ and radius $R$. The domain has solid boundaries; free-slip at the upper surface and no-slip at the base and side, which rotate at a constant rate. There is a prescribed heat flux in the vertical direction which allows the temperature to be decomposed into the form $T=T_{0}(z)+\theta(\mathbf{x}, t)$, where $\theta(\mathbf{x}, t)$ is the deviation from the linear background profile $T_{0}(z)=\left(\mathrm{d} T_{0} / \mathrm{d} z\right) z$. The advantage of this decomposition is that the boundary conditions for a constant vertical heat flux can now be written as $\partial \theta / \partial z=0$ on the upper and lower surfaces. These conditions are all summarised in the schematic of the flow domain in figure 2 .

The reference frame co-rotates with the lower boundary at rate $\boldsymbol{\Omega}$. Assuming an incompressible Boussinesq fluid of bulk density $\rho_{0}$, expansion coefficient $\beta$, kinematic viscosity $\nu$, and thermal diffusivity $\alpha$, the governing equations can be written:

$$
\frac{\mathrm{D} \mathbf{u}}{\mathrm{D} t}=-\frac{1}{\rho_{0}} \nabla p-2 \boldsymbol{\Omega} \times \mathbf{u}+\nu \nabla^{2} \mathbf{u}-\beta \theta \mathbf{g},
$$

and

$$
\frac{\mathrm{D} \theta}{\mathrm{D} t}=\alpha \nabla^{2} \theta+\left|\frac{\mathrm{d} T_{0}}{\mathrm{~d} z}\right| u_{z},
$$

where $\mathbf{g}$ is the gravitational acceleration, and the centrifugal force has been incorporated into the pressure.

Performing a poloidal-azimuthal decomposition on the velocity such that $\mathbf{u}_{p}=\left(u_{r}, 0, u_{z}\right)$ and $\mathbf{u}_{\phi}=\left(0, u_{\phi}, 0\right)$ equation (1) can be re-written [24] as:

$$
\frac{\mathrm{D}}{\mathrm{D} t}\left(\frac{\omega_{\phi}}{r}\right)=\frac{\partial}{\partial z}\left(\frac{\Gamma^{2}}{r^{4}}\right)+\frac{2 \Omega}{r} \frac{\partial u_{\phi}}{\partial z}-\frac{\beta \mathrm{g}}{r} \frac{\partial \theta}{\partial r}+\frac{\nu}{r^{2}} \nabla_{*}^{2}\left(r \omega_{\phi}\right),
$$

and

$$
\frac{\mathrm{D} \Gamma}{\mathrm{D} t}=-2 \Omega r u_{r}+\nu \nabla_{*}^{2} \Gamma,
$$

where $\omega_{\phi}$ is the azimuthal vorticity, $\Gamma=r u_{\phi}$ is the specific angular momentum in the rotating frame, and

$$
\nabla_{*}^{2}=r \frac{\partial}{\partial r}\left(\frac{1}{r} \frac{\partial}{\partial r}\right)+\frac{\partial^{2}}{\partial z^{2}}
$$




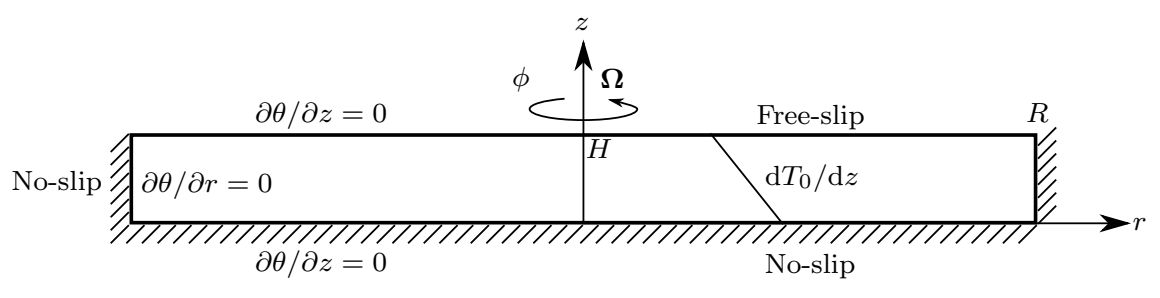

FIG. 2: Schematic of the flow domain.

is the Stokes operator.

Making use of the characteristic velocity scale $U=\sqrt{\mathrm{g} \beta\left(\mathrm{d} T_{0} / \mathrm{d} z\right)} H$ we introduce a Reynolds number for the flow as:

$$
R e=\frac{U H}{\nu} .
$$

The three other dimensionless parameters that control the flow are the Prandtl number, $\operatorname{Pr}=\nu / \alpha$, the Ekman number, $E k=\nu /\left(\Omega H^{2}\right)$, and the aspect ratio $\Lambda=H / R$.

\section{METHODS}

We performed numerical simulations using a finite difference method. Equations (1) and (2) are solved using second-order spatial differences and first-order integration in time from some specified initial condition. The numerical methods are based on those of Harlow and Welch [25], adapted for an axisymmetric coordinate system as was done for the studies of Harlow and Stein [12]. The simulations use a regular staggered mesh of 1000 radial $\times 100$ axial cells. Spatial and temporal resolution studies were performed by increasing and decreasing both the mesh resolution and timestep to ensure the results were converged. We compare our steady results of eye formation to those of Oruba et al. [18] below and find they agree well. Following the approach in [18] we initially chose to keep $\operatorname{Pr}, E k$, and $\Lambda$ all fixed at 0.1 and varied $R e$ as our main parameter of interest. Of course, the Reynolds number is a proxy for the thermal forcing of the cyclone, setting the heat flux through the domain.

The first results were obtained by treating the flow as an initial value problem, integrating in time until a steady solution was obtained. For subsequent runs we took the common approach of incrementally increasing the forcing $(R e)$ and using the end-state of one simulation as the initial condition for the next. Provided the increase in Re between cases is not too large, this allows us to avoid the computational effort associated with calculating the long transients during the initial spin-up.

A number of different parameters were tracked of the course of the simulations as potential ways of characterising the eye. We choose as a diagnostic for the strength of the eye the maximum downwards velocity on the axis. This is the parameter that provided the cleanest time series for measuring variation in the oscillatory cases.

\section{STEADY FLOWS}

Let us begin by considering relatively weak flows that do not display an eye and gradually increase the forcing. These steady state results can be seen in figure 3 where the meridional circulation has been plotted using the Stokes stream function, $\Psi$. In all cases $E k=\operatorname{Pr}=\Lambda=0.1$. At low Reynolds number (figure 3a) a single large meridional convection cell has formed. As Re is increased, the boundary layer at the base intensifies, with strengthening azimuthal vorticity $\omega_{\phi}$. This vorticity is swept up into the bulk flow as it converges towards the rotation axis to form the conical region of negative azimuthal vorticity known as the eyewall.

These results are similar to those of Oruba et al. [18] and we see that as Re is increased further, the eyewall strengthens as the upwards advection of vorticity dominates over diffusion. Eventually Prandtl-Batchelor diffusion of $\omega_{\phi}$ out of the eyewall allows for the formation of an eye. The size and strength of the eye grows as we increase $R e$ and the boundary layer vorticity strengthens. The size of the eye does not vary much between $R e=266$ and $R e=300$ (figures 3d and 3e) but it continues to increase in strength. Clearly there must be a critical Reynolds number at 


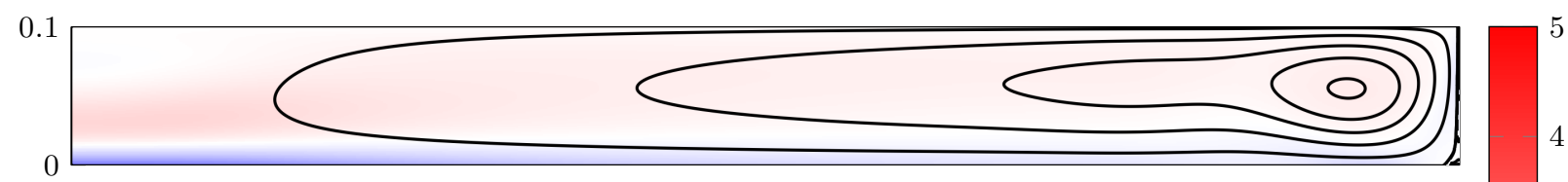

(a) $R e=100$

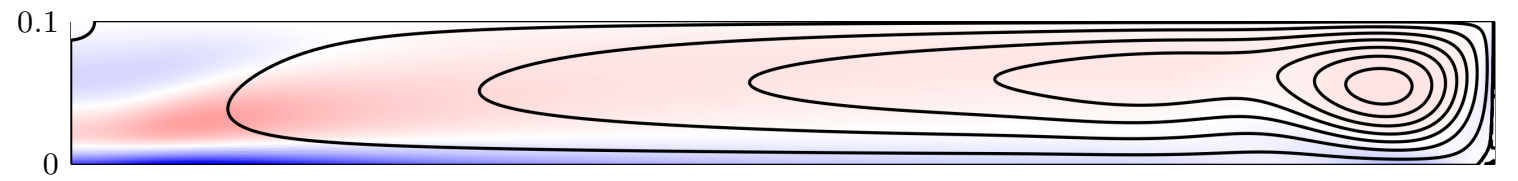

(b) $R e=130$

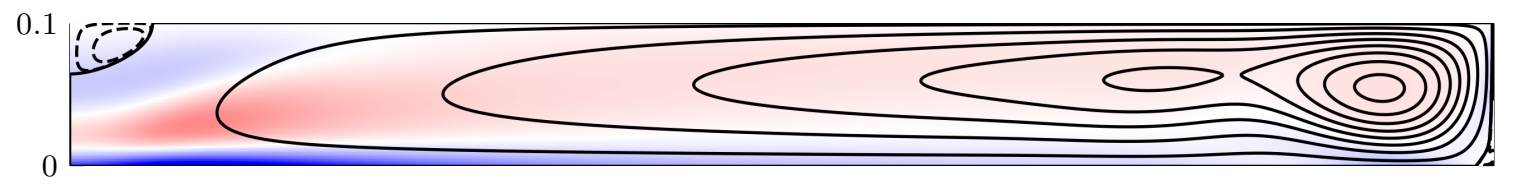

(c) $R e=141$

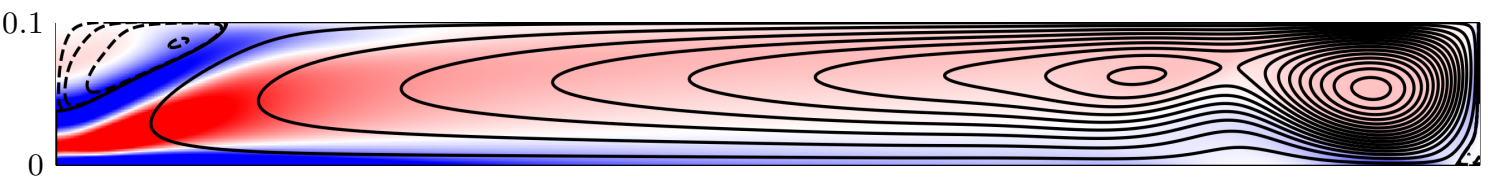

(d) $R e=266$

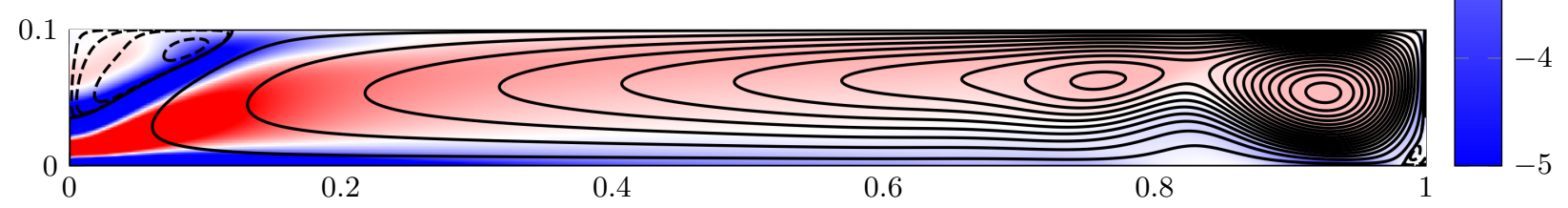

(e) $R e=300$

FIG. 3: Plots of $\omega_{\phi} / r$ overlaid with of meridional streamlines, $\Psi$, for a variety of Reynolds numbers, showing the formation of a steady eye that grows as forcing is increased. In all cases $E k=\operatorname{Pr}=\Lambda=0.1$.

Solid streamlines represent the bulk, clockwise circulation, whilst dashed streamlines indicate anticlockwise motion.

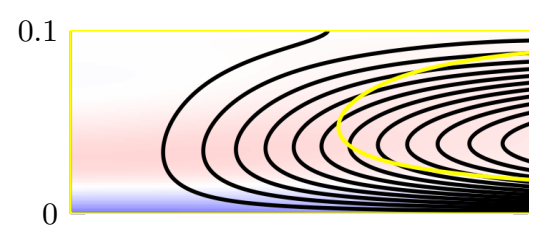

(a) $R e=100$

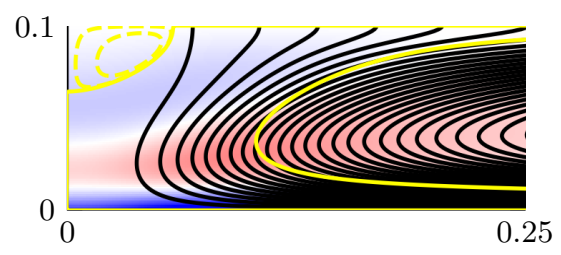

(c) $R e=141$

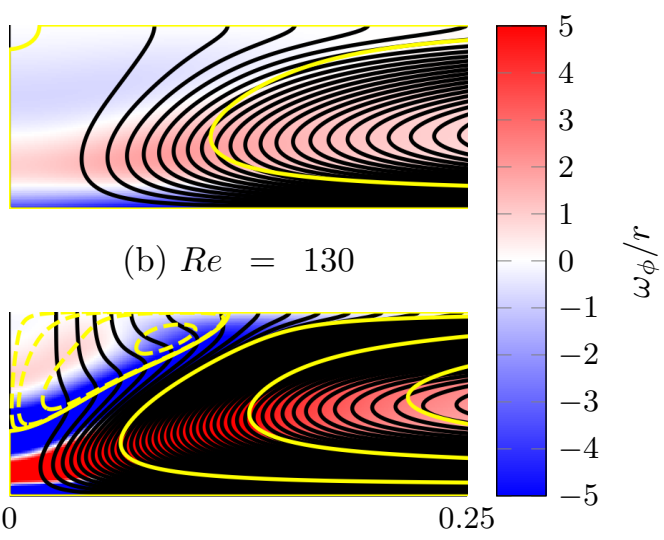

(d) $R e=300$

FIG. 4: Plots of $\omega_{\phi} / r$ overlaid with contours of angular momentum, $\Gamma$, (black), and streamlines (yellow) for a range of Reynolds numbers with $E k=\operatorname{Pr}=\Lambda=0.1$. 
which the steady region of reversed flow - the eye - first forms near the top of the axis. Our results suggest that for an aspect ratio, Prandtl, and Ekman number of 0.1 this critical value is $R e \approx 128$; consistent with [18].

In addition to the streamlines it is instructive to also consider the contours of angular momentum. This will later help us understand the mechanism by which oscillations are driven. These contours are shown in figure 4 near the eye region where the effects of background rotation are weak (see below). Note that, due to the free-slip boundary condition, the contours of angular momentum must meet the upper surface at right angles. Near the base, however, they lie almost parallel to the lower surface due to the material advection of angular momentum by the inflow and the no-slip boundary condition. Diffusion is strong in the lower boundary layer and eyewall, but outside these areas diffusive effects are weaker and we expect the angular momentum contours to more or less follow the streamlines as per equation (4). This is indicated in figure 4a. Close to the axis the meridional flow is weak, and the contours of $\Gamma$ peel away from the streamlines as $\Gamma$ diffuses into the eye in much the same way as $\omega_{\phi}$. As a result the contours of angular momentum in the vicinity of the eyewall straighten, becoming more vertical instead of following the meridional circulation. This can be seen in figures $4 \mathrm{~b}$ and $\mathrm{c}$.

As $R e$ is increased further and a larger eye develops (figure $4 \mathrm{~d}$ ) it can be seen that the contours of $\Gamma$ move further in towards the axis, particularly at the upper levels where the flow in the eye sweeps them inwards. This results in the contours developing a kink as can be seen in figure $4 \mathrm{~d}$. At this point we consider the first term of equation (3), $\partial\left(\Gamma^{2} / r^{4}\right) / \partial z$. The kinking of the contours of $\Gamma$ will give positive axial gradients, and therefore act as a source of positive azimuthal vorticity. This can be seen in figures 3d, 3e and $4 \mathrm{~d}$ where the top left region near the axis has developed positive vorticity. This steady state represents an equilibrium between competing effects; there is the diffusion of negative vorticity into the eye from the eyewall, but the resulting flow acts to sweep the contours of $\Gamma$ inwards to a point at which they become a competing source of positive azimuthal vorticity.

Finally, we consider the angular velocity of the flow in the inertial frame of reference as a combination of the local and background rotation, $\Omega+u_{\phi} / r$. Plotting this in figure 5 , normalised by $\Omega$, we see that the absolute rotation in the inertial frame is large near the axis and in the eye, and weaker towards the outer boundary where it falls to the level of the background rotation. The lower plot shows the $z$-averaged total angular velocity as a function of radius. It is worth taking a moment to note the distinction between angular velocity and $u_{\phi}$; though rotation near the axis is large, the velocity is low, as for a tropical cyclone, peaking around $r=0.1 R$ near the eyewall.

In the next section we show how oscillations develop from this steady flow.
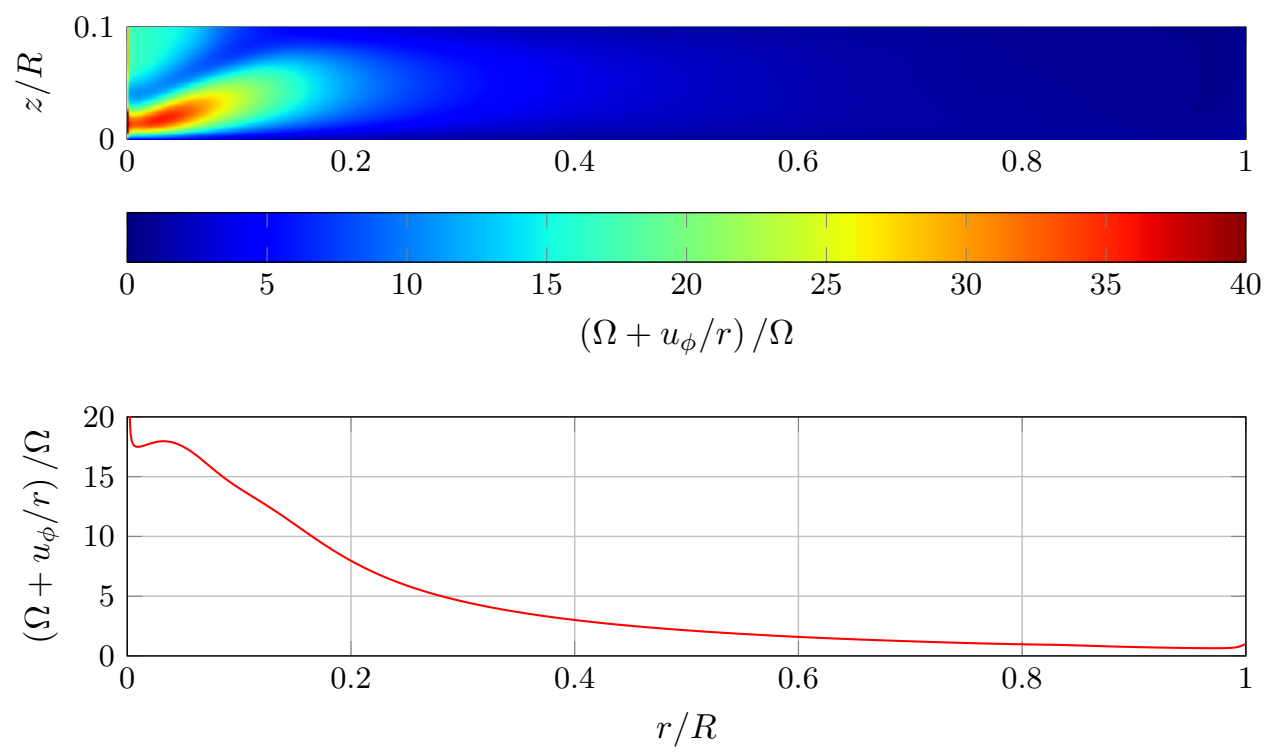

FIG. 5: Normalised total angular velocity for the flow (top) and averaged in the z direction (bottom) $R e=300$.

\section{OSCILLATORY FLOWS}

As the forcing is increased further we observe that, once the Reynolds number exceeds a second critical value, the eye ceases to be steady. The nature of this oscillation is illustrated in figure 6 . It begins with the familiar recirculating eye near the axis inside the eyewall (figure 6a). The centre of the recirculating region (i.e. the region of negative 
$\Psi$ ) then moves radially outwards towards the eyewall (figures $6 \mathrm{~b}$ and c). This reaches an extreme around halfway through the oscillation cycle (figure 6d), where the recirculating region has nearly been pinched off from the axis to exist as an annulus between $r=0.05 R$ and $r=0.10 R$. At this point the reversed flow near the axis strengthens (figures 6e and $\mathrm{f}$ ) expanding to reconnect with the displaced annulus (figure $6 \mathrm{~g}$ ) and returning to the starting state of a large eye filling the region between the eyewall and the axis (figure $6 \mathrm{~h}$ ).

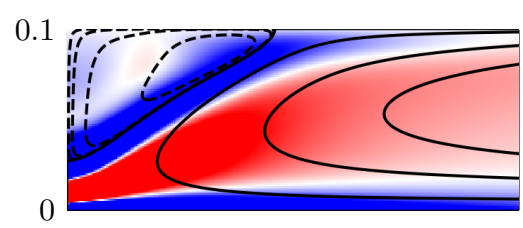

(a) $t / T=0.00$

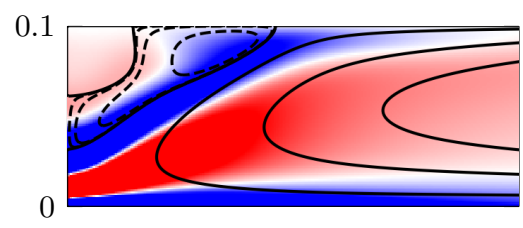

(c) $t / T=0.24$

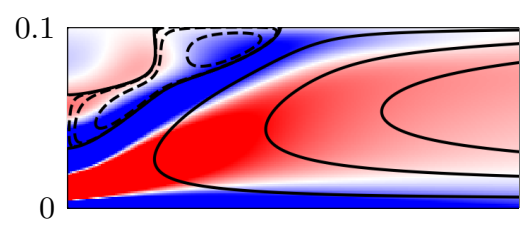

(e) $t / T=0.56$

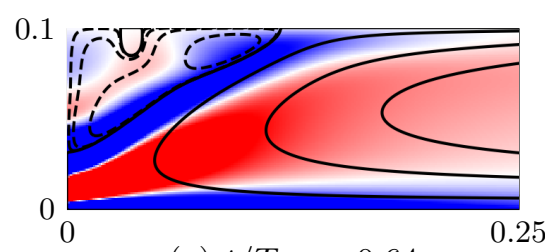

(g) $t / T=0.64$

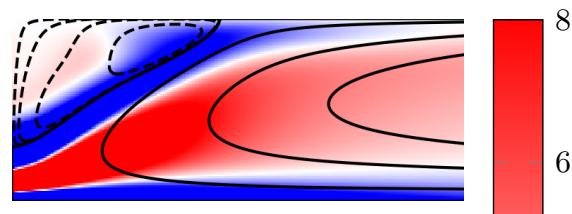

(b) $t / T=0.16$

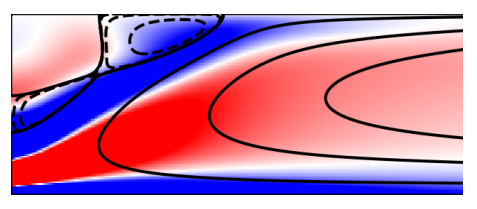

(d) $t / T=0.48$

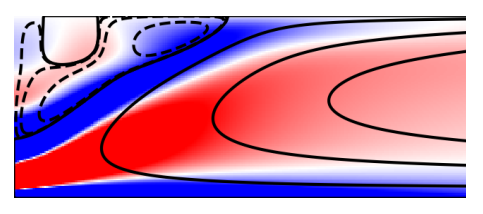

(f) $t / T=0.60$

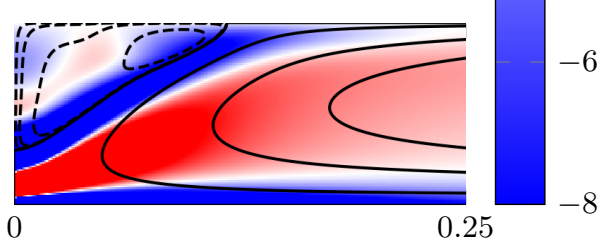

(h) $t / T=0.72$

FIG. 6: A series of plots showing how streamlines and $\omega_{\phi} / r$ vary over one complete oscillation cycle $(T)$ for $E k=\operatorname{Pr}=\Lambda=0.1, R e=400$

It is useful to examine a time series associated with the oscillations. The maximum downwards velocity on the axis follows the spatial oscillations of the eye with the largest magnitude occurring when the eye is full, and the smallest when it is displaced towards the eyewall. The strength of the oscillations can be obtained from the time series once it reaches a steady amplitude. This is shown in figure 7 . The Fourier transform of the time series yields the frequency of the oscillations (figure 8).

At yet higher values of Re the oscillations cause the eyewall to become significantly distorted. As it snaps back to a fully formed state, a region of negative vorticity is pinched off near the top of the domain and is carried radially outwards along the upper surface. This behaviour is illustrated in figure 9, which shows streamlines for the duration of one cycle. We note that the oscillations we observe here display qualitative similarities to those seen by Harlow and Stein [12] for columnar vortices. Eventually, as forcing continues to increase, the system becomes increasingly aperiodic with the peaks in the Fourier transforms becoming less clear, and the noise elsewhere in the spectrum increasing. This corresponds to the oscillations becoming increasingly complex with fluctuations in amplitude. This can clearly be seen in the Fourier transform of the at high Reynolds number in figure 8d.

We find that the critical Reynolds number at which oscillations in the eye begin, for $\operatorname{Pr}, E k$, and $\Lambda$ of 0.1 , is $R e_{c} \approx 398$. The nature of this transition can be examined by plotting the amplitude of the oscillations against $\left(R e-R e_{c}\right)$ as in figure 10. The amplitude scales with $\left(R e-R e_{c}\right)^{1 / 2}$ up to a value of $\left(R e-R e_{c}\right) / R e_{c}=0.2$. Beyond this point higher order effects come in to play. This is typical behaviour of the Landau equation, with the transition 


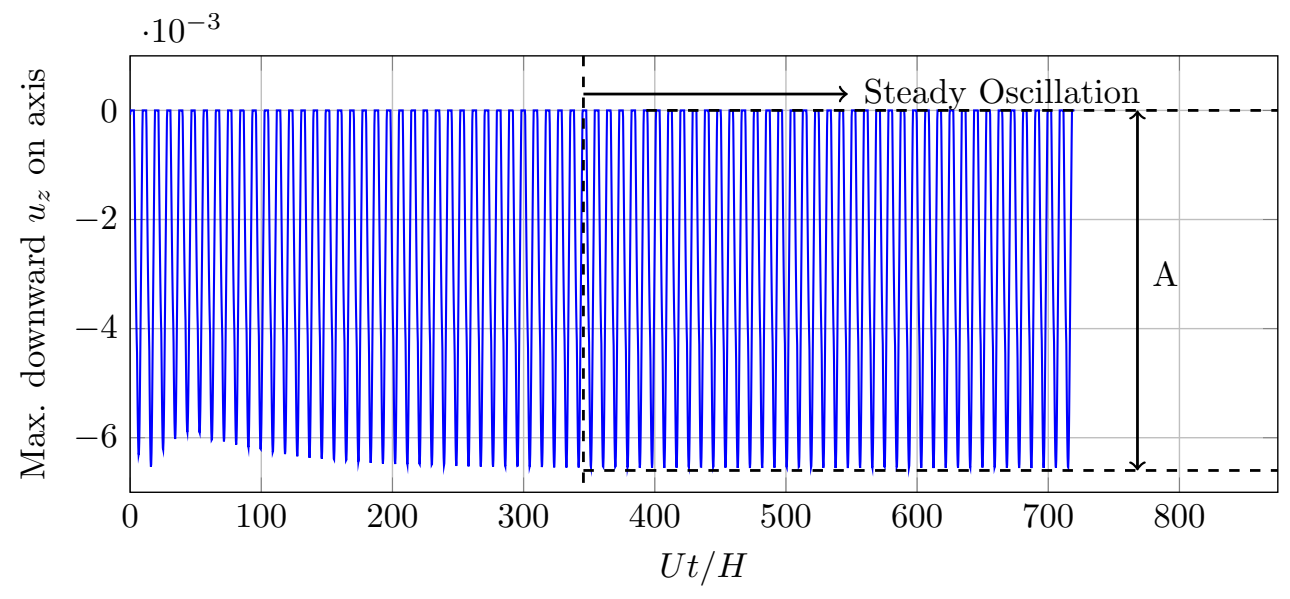

FIG. 7: Time series for the case $R e=480$. Time is scaled by $H / U$.

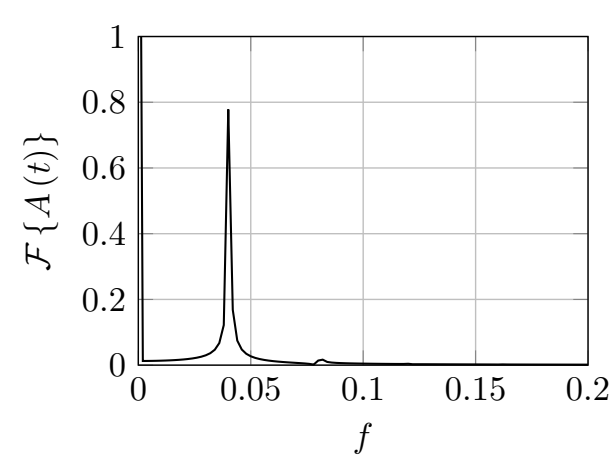

(a) FFT for $R e=400$

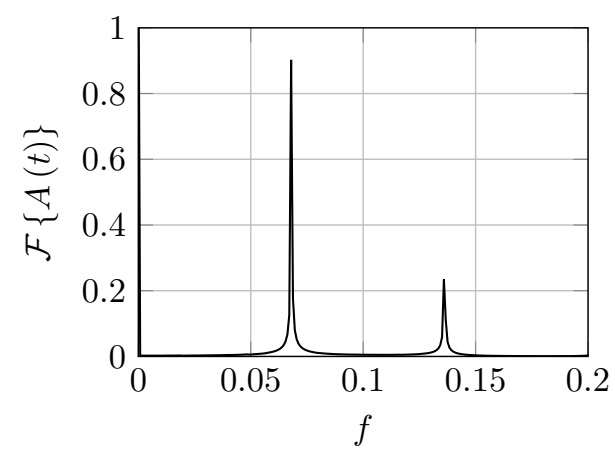

(c) FFT for $R e=650$

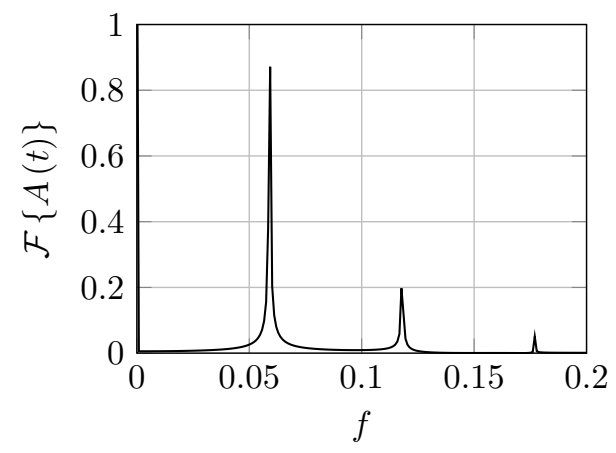

(b) FFT for $R e=550$

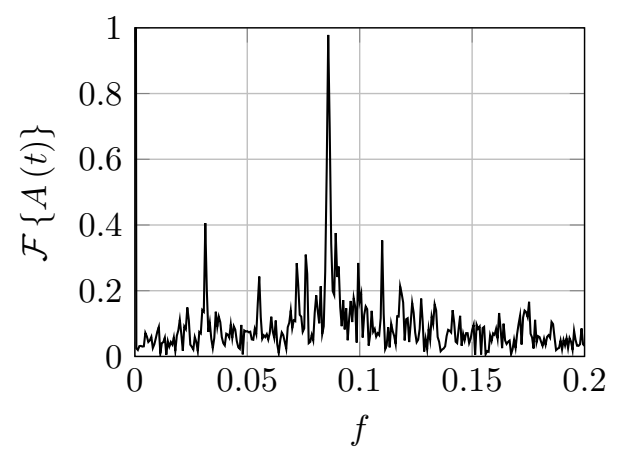

(d) FFT for $R e=750$

FIG. 8: Fourier transforms of the $u_{z}$ time series for different levels of forcing $(R e)$.

to an oscillatory state being a supercritical Hopf bifurcation.

It is common practice for rotating flows displaying a bifurcation, such as Taylor-Couette flow, to examine the dependence upon the rotation rate of the system. To this end we performed simulations at a variety of $E k$ around $R e_{c}$ to see how the critical value for transition changes. The results of this can be seen in figure 11. It was observed that the critical Reynolds number at which oscillations begin increases with $E k$ until around $E k=0.125$. Beyond this it appears that only steady eyes can exist. This is because oscillations are viscously damped. Increasing $E k$ further we found that no eye formed for $E k>0.23$, consistent with [19]. For $E k$ less that 0.1, we find that the critical Reynolds number for oscillations falls dramatically as viscous effects reduce. 


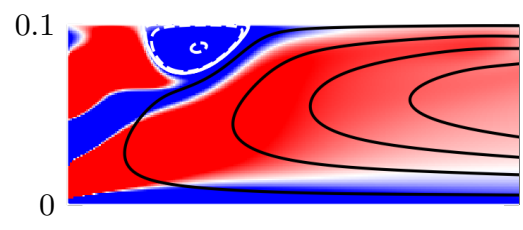

(a) $t / T=0.00$

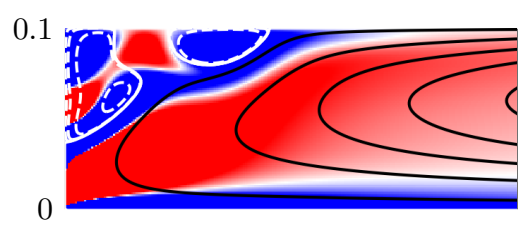

(c) $t / T=0.27$

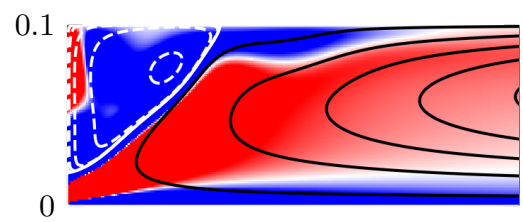

(e) $t / T=0.54$

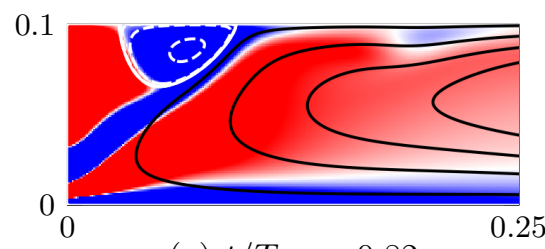

(g) $t / T=0.82$

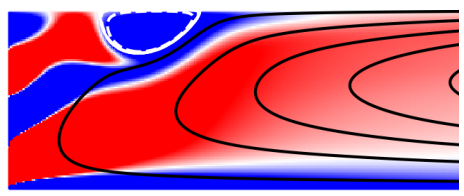

(b) $t / T=0.14$

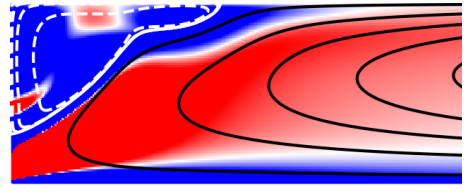

(d) $t / T=0.41$

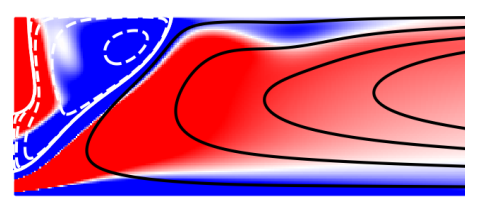

(f) $t / T=0.68$

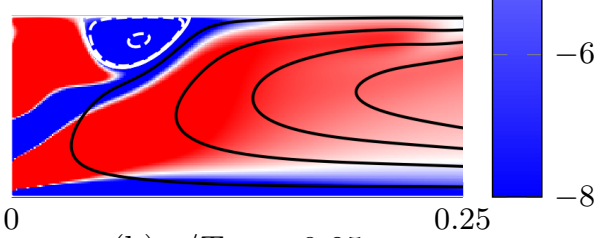

(h) $t / T=0.95$

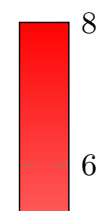

4

2

$0 \stackrel{2}{3}$

$-2$

$-4$

$-6$ $-8$

FIG. 9: A series of plots showing how streamlines and $\omega_{\phi} / r$ vary over one complete oscillation cycle $(T)$ for $E k=\operatorname{Pr}=\Lambda=0.1, R e=650$

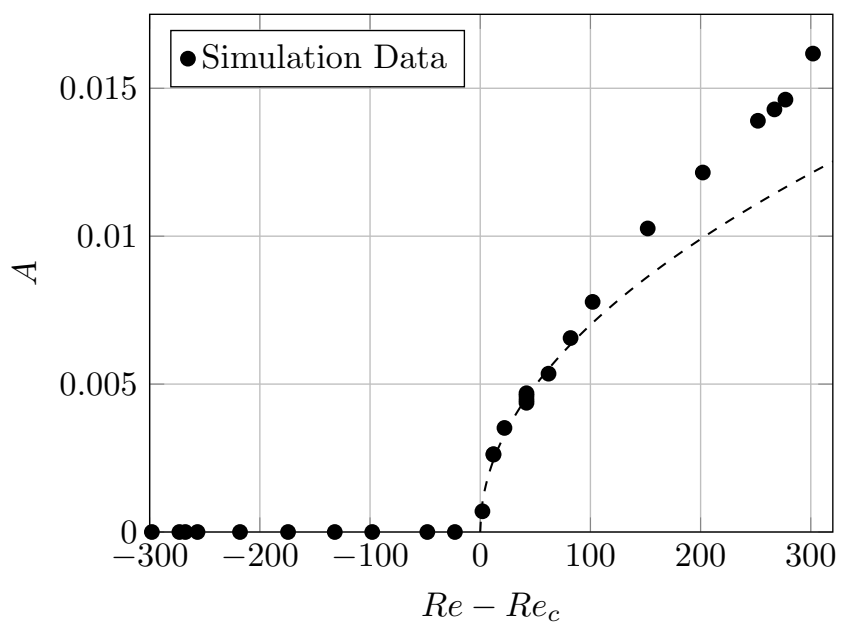

FIG. 10: Bifurcation diagram for the oscillating eye. The dashed line is proportional to $\left(R e-R e_{c}\right)^{1 / 2}$. 


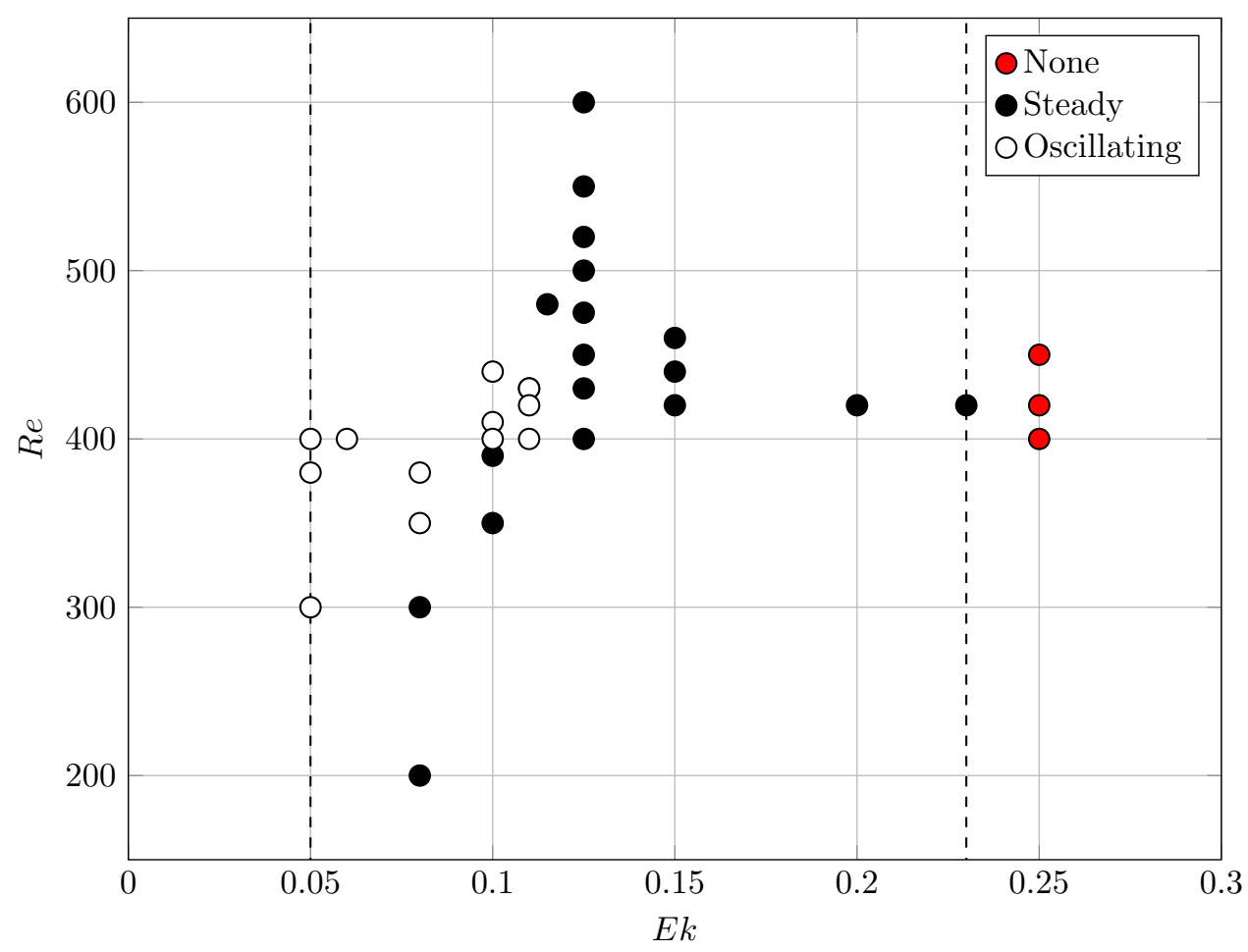

FIG. 11: Regime diagram for eyes showing $R e$ plotted against $E k$ for the case $\Lambda=\operatorname{Pr}=0.1$.

\section{THE OSCILLATION AS A TRAPPED INERTIAL WAVE}

We now seek to describe a physical mechanism by which this oscillation occurs. Perturbations to an incompressible fluid rotating about a central axis are subject to restoring action through the Coriolis force when viewed in the rotating reference frame. This makes such a fluid a wave-bearing system, capable of supporting oscillations known as inertial waves. This phenomenon is described at length in Greenspan [26] and Davidson [24]. Closed wave-bearing systems often display natural frequencies and associated mode shapes. It is possible, therefore, that the observed behaviour of the eye might be explained by the presence of a 'trapped' or 'standing' inertial wave.

To examine this claim it is necessary to probe the dynamics of the eye a little more closely to see whether it might be capable of sustaining inertial waves. We saw in figure 5 that the angular velocity at the centre of the vortex was much higher than the background rotation. We now look more specifically at the rotation in the eye region. Averaging $u_{\phi}$ and $\Psi$ in time across one complete cycle we define the time-averaged eye as the region in which $\Psi_{\text {avg. }}<0$. We then average in the $z$-direction over the eye to examine how $u_{\phi}$, averaged in both time and $z$, varies with $r$, as shown in figure 12. We see that the mean angular velocity in the eye is significantly larger than the background rotation.

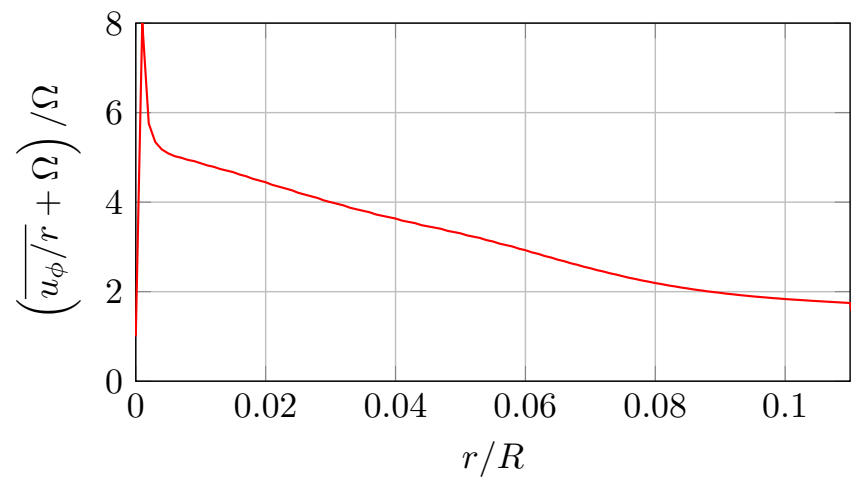

FIG. 12: Plot of variation of time and z- average of $\left(u_{\phi} / r+\Omega\right) / \Omega$ in the eye, $R e=480$. 


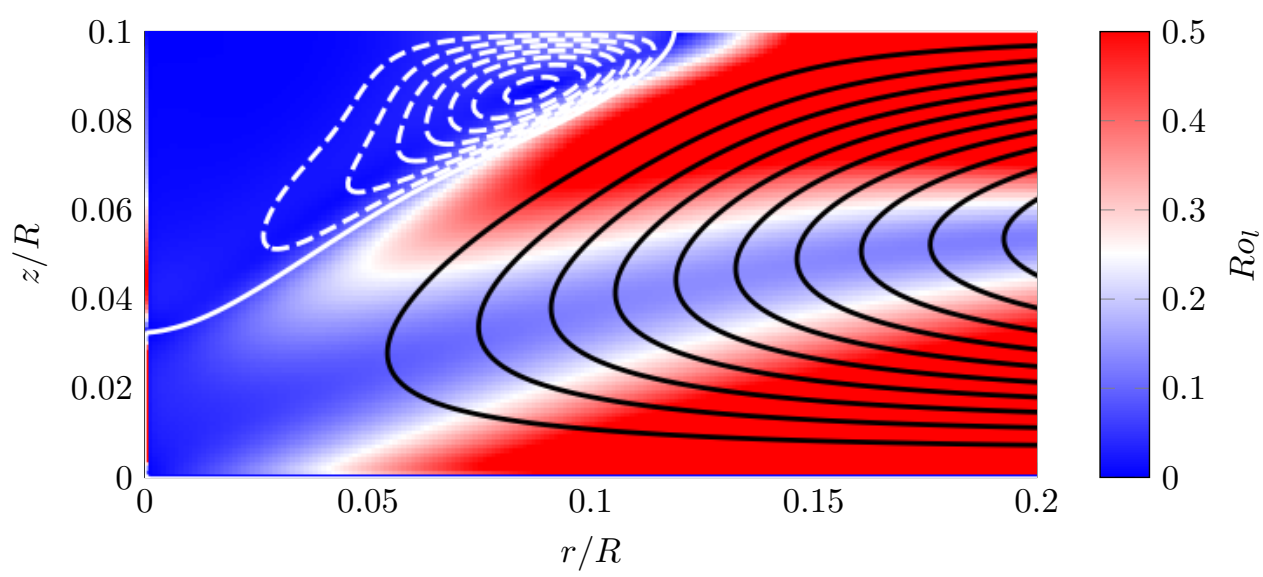

FIG. 13: $R o_{l}$ in the eye (top) and bulk (bottom) for the case $R e=350$.

We can introduce a local Rossby number for the flow as:

$$
R o_{l}=\frac{\sqrt{\mathbf{u}_{p}^{2}}}{2 H \cdot\left(u_{\phi} / r+\Omega\right)},
$$

where $\mathbf{u}_{p}$ is the poloidal velocity. This approach was first suggested by Morton [1] who notes that, for flows with intense swirl, the effects of rotation from the local flow could well be more significant than those due to any background rotation. Figure 13 shows $R o_{l}$ for a Reynolds number just below that which is critical value for oscillations to occur. We can see that $R o_{l}$ is small in the vicinity of the eye, and larger in the bulk of the flow, as might be expected. A closer look at the eye region shows a clear change in $R o_{l}$ that is bounded by the $\Psi_{\text {avg }}=0$ streamline at the edge of the eyewall. It is well known [24] that a requirement for inertial waves to be present is small Rossby number, i.e. rapid rotation. A study by Staplehurst et al. [27] found inertial waves emerging in a rotating fluid for a Rossby number below 0.4. Examining figure 13 we see that $R o_{l}$ in the eye is well below this, and therefore may be capable of supporting inertial oscillations.

Henderson and Aldridge [28] and Beardsley [29] study inertial waves in a frustum (a truncated cone), the approximate shape of the eye. They both record a base natural frequency of $\varpi / \Omega=1.12$ for a given aspect ratio, noting that the mode shapes in the frustum appear to be a perturbed version of those for a cylinder. They also both observe the absence of a modified first mode for a cylinder $(r, \phi, z)=(1,0,1)$ in their results, with the lowest mode observed both numerically and experimentally for a frustum being $(1,0,2)$. It is possible to calculate an analytical solution for inertial waves in a cylinder for which an aspect ratio of $R / H=1$ gives a frequency of $\varpi / \Omega=1.26$ for the first mode $(1,0,1)$, and 1.71 for the second axial mode $(1,0,2)$. Comparing the natural frequency of a cylinder and frustum of the same aspect ratio, we find that $\varpi / \Omega=1.42$ for mode $(1,0,2)$ in a cylinder compared to 1.12 for the frustum discussed above. This provides some indication of how shape changes frequency. These values are all consistent with the general result for inertial waves that the frequency $\varpi$ must lie in the range 0 to $2 \Omega$, where $\Omega$ is the rotation rate. Taking an estimate of the average angular velocity in the eye, we obtain an estimate of $\varpi / \Omega=1.54$ for our $R e=400$ simulation. This analysis can be repeated for other values of $R e$ with the results shown in figure 14 . We see that all of the frequencies for eye oscillations lie within the required bound for inertial waves, with values around those expected for a cylinder or frustum.

We return at this point to discuss further the observations of high frequency oscillations in tropical cyclones by Chen et al. [17]. They report fluctuations in a number of parameters in the eye region with a typical period of around 2 hours (between 90 and 150 minutes). One of these parameters is the water vapor convection which is directly related to the vertical motion. Figure 15 shows the variation of this property in the eye region of a simulated Typhoon Hagupit (one of the cyclones in which high frequency oscillations were observed) as a function of height and time. It can be seen that the oscillations occur over the height of the cyclone with a period of approximately 2 hours. If we adopt a similar approach to the one taken for our simulations we can estimate the local rotation rate in the core from the reported maximum windspeed. This is around $50 \mathrm{~m} \mathrm{~s}^{-1}$ at a radius of approximately $50 \mathrm{~km}$ which gives an angular velocity of $3.6 \mathrm{rad} \mathrm{hr}^{-1}$. For oscillations with a period of around 2 hours this gives an estimation of $\varpi / \Omega=0.9$ which is of the order observed in our simulations, and below the upper bound of 2 for inertial waves. It is possible, therefore, that these oscillations are caused by trapped inertial waves, although this hypothesis requires further investigation.

Finally we consider the contours of $\Gamma$ in the eye. These are plotted over the course of one oscillation in figure 


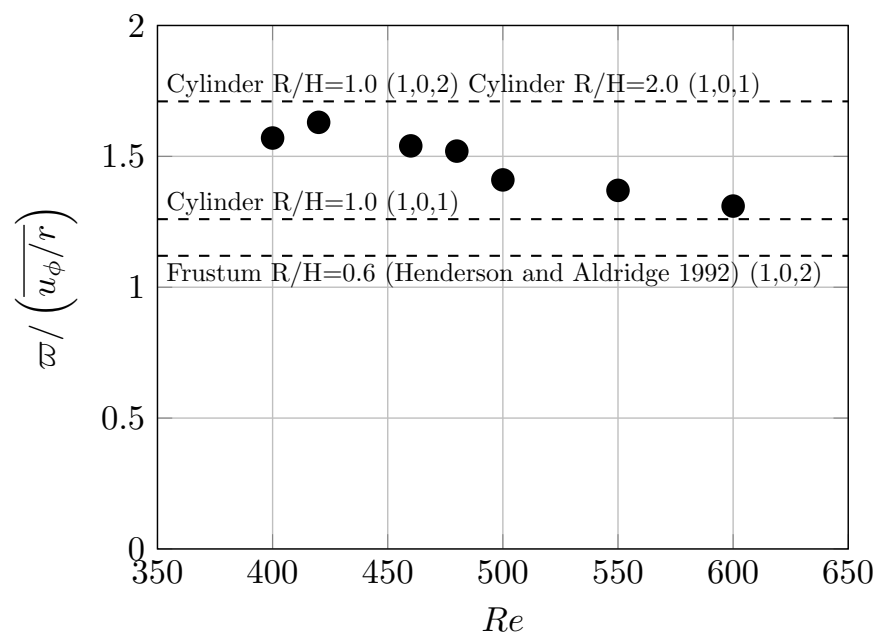

FIG. 14: $\varpi / \Omega$ for the oscillations vs. Re, along with selected values for a cylinder and frustum for comparison

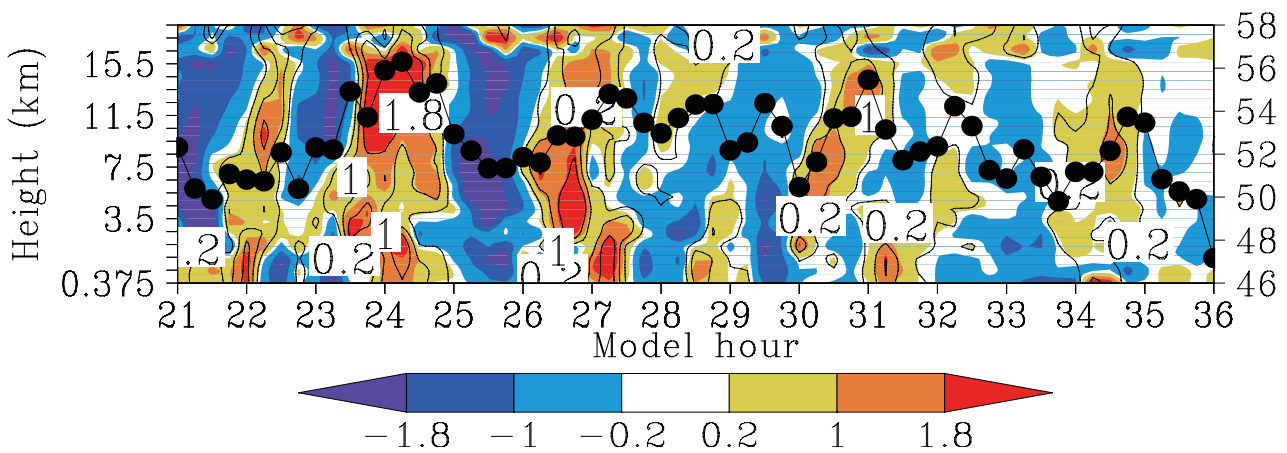

FIG. 15: Figure 11a from Chen et al. [17] showing oscillations in a simulated Typhoon Hagupit. Colour shows the time-height variation of water vapor convection whilst the black line shows the maximum windspeed in $\mathrm{ms}^{-1}$ (scale on right). Reproduced with permission.

16. In the bulk flow the contours broadly follow the streamlines, as expected from equation (4). At the top of the domain they are perpendicular to the upper boundary due to the free-slip boundary condition. In the eye, however, the contours deviate from the streamlines. It can be seen that over the course of one oscillation the contours go from initially being close to vertical to lean to the left, then to the right, before returning to their original state in a kind of 'sloshing' motion. Figure 17 shows the same phenomenon, but at a higher level of forcing where the motion of the contours is more noticeable. Figure 18 shows $\omega_{\phi} / r$ and the contours of $\Gamma$ for the first mode of a standing inertial wave in a cylinder. Comparing the eye and the cylinder we can see that the two follow qualitatively the same pattern. This is as we might expect given the observations of Henderson and Aldridge [28] and Beardsley [29] that standing waves in a frustum are perturbed versions of those in a cylinder.

Equation (3) can provide perhaps a more physical understanding of the nature of oscillations. The 'sloshing' of the contours of $\Gamma$ back and forth leads to a variation of $\omega_{\phi} / r$ as a result of the $\partial \Gamma / \partial z$ term. Both Rotunno [30] and Davidson [24,31] provide good discussions of how this vortex stretching generates azimuthal vorticity. The induced variation in $\omega_{\phi} / r$ can be seen in figures 6,16 , and 17 . The process can be summarised as follows: the flow in the fully formed eye sweeps the contours of $\Gamma$ radially inwards at the upper levels, causing them to slant to the left. This positive axial variation in $\Gamma$ generates positive $\omega_{\phi}$ that counteracts the flow in the eye causing it to be swept outwards towards the eyewall. The flow again carries with it the angular momentum causing a rightward slant to the contours of $\Gamma$. This in turn generates negative $\omega_{\phi}$ and the eye is restored before the process then repeats. 


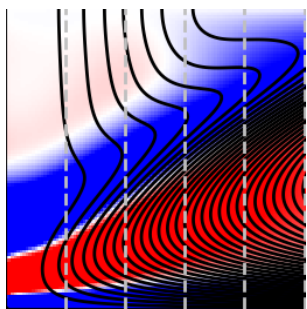

(a) $t / T=0.00$

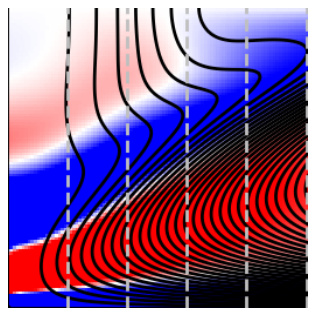

(b) $t / T=0.32$

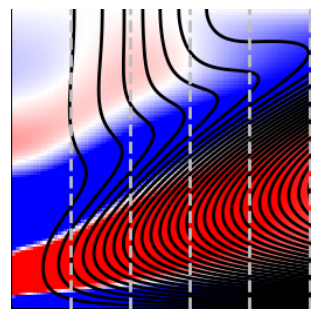

(c) $t / T=0.44$

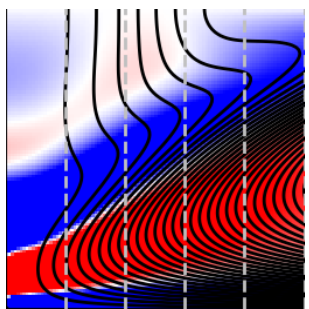

(d) $t / T=0.56$

FIG. 16: A series of plots showing how contours of $\Gamma$ evolve in the eye over one cycle $(T)$ for $E k=\operatorname{Pr}=\Lambda=0.1, \operatorname{Re}$ $=400$. Colour $=\omega_{\phi} / r$.

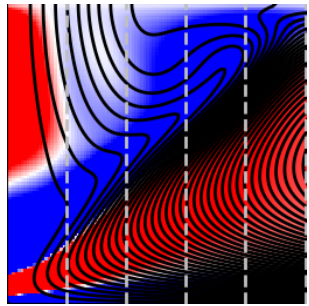

(a) $t / T=0.00$

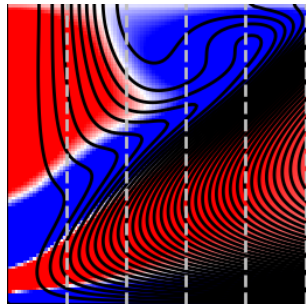

(b) $t / T=0.08$

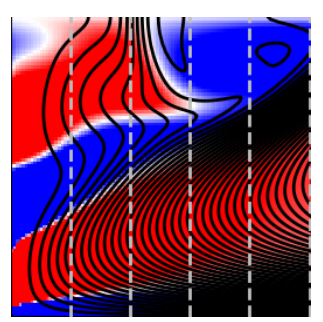

(c) $t / T=0.24$

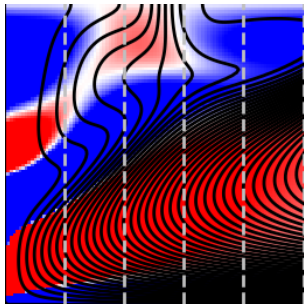

(d) $t / T=0.40$

FIG. 17: A series of plots showing how contours of $\Gamma$ evolve in the eye over one cycle $(T)$ for $E k=\operatorname{Pr}=\Lambda=0.1, \operatorname{Re}$ $=600$. Colour $=\omega_{\phi} / r$.

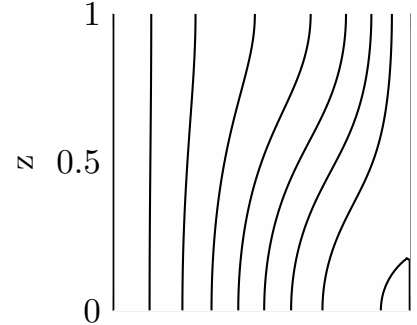

(a) $t / T=0.00$

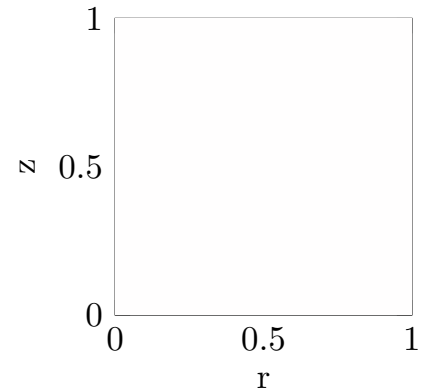

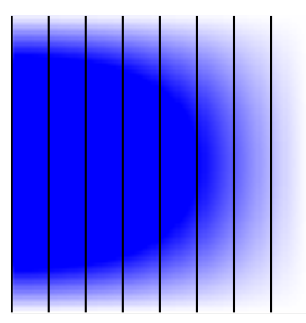

(b) $t / T=0.25$

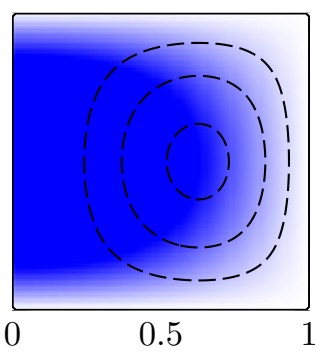

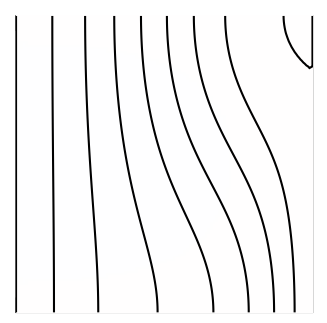

(c) $t / T=0.50$

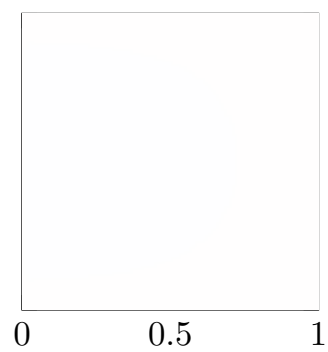

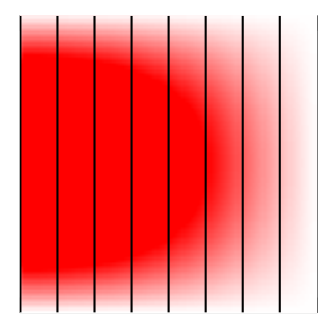

(d) $t / T=0.75$

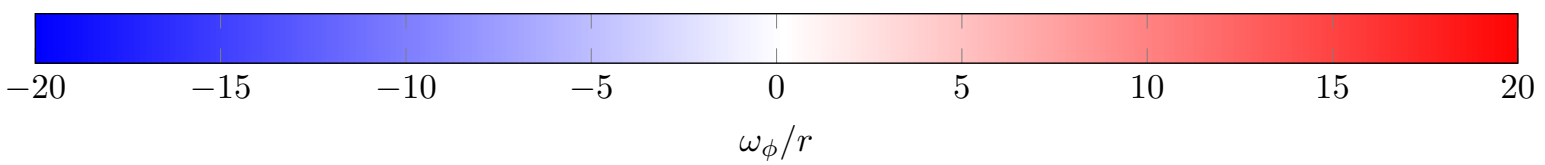

FIG. 18: A series of plots showing how contours of $\Gamma$ (top) and streamlines (bottom) evolve over one cycle (of period $\mathrm{T})$ for a standing inertial wave in a cylinder. 


\section{CONCLUSIONS}

We have examined the system of rotating convection first proposed by Oruba et al. [18]. The resulting flow may form an eye at the centre of a cyclonic vortex. By increasing the forcing we observed that the the system can undergo a bifurcation from a state with a steady eye to one in which the eye oscillates. For an aspect ratio, Ekman, and Prandtl numbers of 0.1 we find that the critical Reynolds number at which this transition occurs is 398 . Examining how the amplitude of the oscillations vary with increased forcing we conclude that this bifurcation takes the form of a supercritical Hopf bifurcation. We examine the dependence on Ekman number, finding that there is an upper limit beyond which only steady eyes are observed. As the Ekman number is decreased the critical Reynolds number for oscillation falls.

Examining the nature of the oscillations we propose that the behaviour results from a trapped inertial wave at the centre of the vortex. The frequency of the oscillations falls within the expected range for inertial waves, and the motions in the eye display clear similarities to those of a standing inertial wave in a cylinder.

\section{ACKNOWLEDGMENTS}

The authors would like to thank Dr. Shumin Chen for helpful correspondence. This work was undertaken with funding from an EPSRC doctoral studentship.

[1] B. R. Morton, "Geophysical vortices," Progress in Aerospace Sciences 7, 145-194 (1966).

[2] P. C. Sinclair, "The lower structure of dust devils," Journal of the Atmospheric Sciences 30, 1599-1619 (1973).

[3] J. H. Golden, "The life cycle of Florida Keys' waterspouts. I," Journal of Applied Meteorology 13, 676-692 (1974).

[4] J. T. Snow, "A review of recent advances in tornado vortex dynamics," Reviews of Geophysics 20, 953-964 (1982).

[5] K. Emanuel, "Tropical cyclones," Annual review of earth and planetary sciences 31, 75-104 (2003).

[6] R. P. Pearce, "Why must hurricanes have eyes?" Weather 60, 19-24 (2005).

[7] R. P. Pearce, "Comments on "Why must hurricanes have eyes? -revisited"," Weather 60, 329-330 (2005).

[8] R. K. Smith, "Why must hurricanes have eyes?"-revisited," Weather 60, 326-328 (2005).

[9] R. K. Smith, "Tropical cyclone eye dynamics," Journal of the Atmospheric Sciences 37, 1227-1232 (1980).

[10] H. E. Willoughby, "Tropical cyclone eye thermodynamics," Monthly weather review 126, 3053-3067 (1998).

[11] R. P. Pearce, "An axisymmetric model of a mature tropical cyclone incorporating azimuthal vorticity," Quarterly Journal of the Royal Meteorological Society 130, 259-293 (2004).

[12] F. H. Harlow and L. R. Stein, "Structural analysis of tornado-like vortices," Journal of the Atmospheric Sciences 31, 2081-2098 (1974).

[13] R. Rotunno, "Numerical simulation of a laboratory vortex," Journal of the Atmospheric Sciences 34, 1942-1956 (1977).

[14] R. Rotunno, "A study in tornado-like vortex dynamics," Journal of the Atmospheric Sciences 36, 140-155 (1979).

[15] T. Maxworthy, "On the structure of concentrated, columnar vortices," Astronautica Acta 17, 363-374 (1972).

[16] D. Castaño, M. C. Navarro, and H. Herrero, "Evolution of secondary whirls in thermoconvective vortices: Strengthening, weakening, and disappearance in the route to chaos," Physical Review E 93, 013117 (2016).

[17] S. Chen, Y. Lu, W. Li, and Z. Wen, "Identification and analysis of high-frequency oscillations in the eyewalls of tropical cyclones," Advances in Atmospheric Sciences 32, 624-634 (2015).

[18] L. Oruba, P. A. Davidson, and E. Dormy, "Eye formation in rotating convection," Journal of Fluid Mechanics 812, 890-904 (2017).

[19] L. Oruba, P. A. Davidson, and E. Dormy, "Formation of eyes in large-scale cyclonic vortices," Phys. Rev. Fluids 3, 013502 (2018).

[20] R. K. Smith and M. T. Montgomery, "Hurricane boundary-layer theory," Quarterly Journal of the Royal Meteorological Society 136, 1665-1670 (2010).

[21] G. I. Taylor, "Stability of a viscous liquid contained between two rotating cylinders," Philosophical Transactions of the Royal Society of London. Series A, Containing Papers of a Mathematical or Physical Character 223, 289-343 (1923).

[22] H. Bénard, "Les tourbillons cebullaires dans une nappe liquide," Revue générale des Sciences pures et appliquées 11, 1261-1274 (1900).

[23] T. B. Benjamin, "Bifurcation phenomena in steady flows of a viscous fluid. I. theory," Proceedings of the Royal Society of London A: Mathematical, Physical and Engineering Sciences 359, 1-26 (1978).

[24] P. A. Davidson, Turbulence in rotating, stratified and electrically conducting fluids (Cambridge University Press, 2013).

[25] F. H. Harlow and J. E. Welch, "Numerical calculation of time-dependent viscous incompressible flow of fluid with free surface," Physics of Fluids 8, 2182 (1965).

[26] H. P. Greenspan, The theory of rotating fluids (Cambridge University Press, 1968). 
[27] P. J. Staplehurst, P. A. Davidson, and S. B. Dalziel, "Structure formation in homogeneous freely decaying rotating turbulence," Journal of Fluid Mechanics 598, 81-105 (2008).

[28] G. A. Henderson and K. D. Aldridge, "A finite-element method for inertial waves in a frustum," Journal of Fluid Mechanics 234, 317-327 (1992).

[29] R. C. Beardsley, "An experimental study of inertial waves in a closed cone," Studies in Applied Mathematics 49, 187-196 (1970).

[30] R. Rotunno, "Vorticity dynamics of a convective swirling boundary layer," Journal of Fluid Mechanics 97, 623-640 (1980).

[31] P. A. Davidson, "The interaction between swirling and recirculating velocity components in unsteady, inviscid flow," Journal of Fluid Mechanics 209, 35-55 (1989). 